\title{
Reachability Deficits in Quantum Approximate Optimization of Graph Problems
}

\author{
V. Akshay, H. Philathong, I. Zacharov, and J.D. Biamonte \\ Skolkovo Institute of Science and Technology, 3 Nobel Street, Moscow, Russia 121205
}

The quantum approximate optimization algorithm (QAOA) has become a cornerstone of contemporary quantum applications development. Here we show that the density of problem constraints versus problem variables acts as a performance indicator. Density is found to correlate strongly with approximation inefficiency for fixed depth QAOA applied to random graph minimization problem instances. Further, the required depth for accurate QAOA solution to graph problem instances scales critically with density. Motivated by Google's recent experimental realization of $\mathrm{QAOA}$, we preform a reanalysis of the reported data reproduced in an ideal noiseless setting. We found that the reported capabilities of instances addressed experimentally by Google, approach a rapid fall-off region in approximation quality experienced beyond intermediate-density. Our findings offer new insight into performance analysis of contemporary quantum optimization algorithms and contradict recent speculation regarding low-depth QAOA performance benefits.

\section{Introduction}

Quantum approximate optimization (QAOA) is the most studied gate-based approach towards quantum enhanced optimization. Google's Sycamore quantum processor was recently used to demonstrate QAOA applied to graph minimization problems [1]. This recent study [1] follows Sycamore's demonstration of computational capabilities surpassing those of classical supercomputers for specific sampling tasks [2].

\footnotetext{
V. Akshay: akshay.vishwanathan@skoltech.ru, http://quantum.skoltech.ru
}

Such contemporary milestones have dramatically increased interest in harnessing quantum processors for more practical means. Towards this goal, recent experimental demonstrations of quantum algorithms also include quantum chemistry [3, 4], machine learning [5, 6], simulation of condensed matter systems $[7,8]$ as well as discrete optimization $[2,9,10]$. While the ultimate prospects of quantum enhanced algorithms is tantalizing, the computational capacity of existing and near term applications remains unclear.

Recently Google's Sycamore quantum processor, which is based on fifty four superconducting qubits, has demonstrated promising results related to quantum enhanced discrete optimization (via QAOA). The authors [1] experimentally realized QAOA for three classes of graph optimization problems. Such problems assign energy to graphs, which depend on nodes - represented as qubits - taking binary values. The experiments minimized this energy for three classes of graphs up to twenty three nodes [1].

QAOA is an instance of the class of variational quantum algorithms. Variational algorithms vary over a family of parameterized quantum states to minimize an objective function calculated from easy to measure observables. Proposed primarily in $[11,12]$, the variational approach is in theory equivalent to the quantum circuit model under a polynomial (Karp) reduction [13]. Hence, the variational approach is as powerful as a general quantum computer. However only restricted forms - which require significant classical resources in the optimization step - can currently be realized in practice.

Indeed, the advantage of the variational approach is also its weakness. A classical optimization loop iteratively adjusts a quantum state prepared by an adjustable quantum circuit (an ansatz). High depth circuits of fixed structure have been proven to represent universal resources for quantum computation $[14,15]$ and hence can 
emulate the gate model piece-wise. However, higher depth circuits demand a significant optimization task to be performed on a classical computer. In contrast, while reasonably short depth circuits have shown noise-free quantum advantage [16-21], less remains known about the depth of circuits required to enable practical advantage using noisy circuits.

Changing to a state dependent picture and extending recent results related to satisfiability [22], here we propose an order parameter which correlates with the performance of QAOA. In particular, we consider the ratio of graph edges to graph nodes (called graph density). Though a careful comparison, we empirically observe the following findings. (i) Approximation inefficiency, characterised by metrics such as success probability, show an inverse dependence on density of randomly generated graph instances. (ii) Longer depth circuits become a necessity for increasingly accurate approximations and this depth correlates with increasing density. Based on these results we, observe instances addressed in Google's experimental demonstration to represent a statistically narrow subset of random instances having densities that are at the edge of a fall-off region.

Contemporary demonstrations of QAOA serve as benchmarks to enable practically relevant demonstrations of optimization and other quantum algorithms [10, 23-27]. In this regard, our results illuminate the first QAOA performance indicators.

\section{Results}

Google considered up to a depth-five QAOA ansatz on three separate classes of graph problems [1]. Under experimental noise, Google was able to realize functional depth-three ansatz performance whereas depths four and five were critically limited by noise. The depth three ansatz involves classical optimization over only six real parameters, three of which are constrained in the interval $[0, \pi)$ whereas the remaining three are in $[0,2 \pi)$.

We reproduced Google's ideal (noiseless) data using exact numerical emulations (see Fig. 1). Further, we show that QAOA on random graph instances generated at varying densities exhibit strong density dependent performance. Although higher depths achieve better performance, the density dependence is still exhibited (see Fig. 2). Importantly, higher depths necessitate increasingly more parameters to be optimized in the classical step.

Based on the comparison of random graphs at approximately the same densities with the restrictive graph instances Google considered, we do not observe any topology dependent performance bias that can be attributed statistical relevance. This finding suggests density - albeit a coarse graining - is the salient limiting performance indicator for fixed depth QAOA (see Fig. 3).

With density as an order parameter, we find that solutions of random instances generated below densities of 0.5 to show the best performance independent of the number of nodes. Beyond density 0.5 , we find the performance to strongly depend on density for fixed number of nodes. In particular, we observe a sharp fall-off behaviour in performance which appears to saturate logistically beyond density 1.5. It is under this new outlook Google's instances are compared and contrasted (see Fig. 4 and Fig. 5).

Firstly, the authors [1] consider the HardwareGrid problem wherein the generated random instances are native to the inherent hardware connectivity layout of Google's Sycamore chip (sometimes called, interaction graph). Here the instances generated by varying the number of nodes $(n)$ which we found to appear at densities between 1.0 and 1.5. Our exact simulation of depth three QAOA suggests the success probability (or the state overlap) to worsen with the number of nodes (increasing density), achieving a meager 0.07 for nodes $n=20$ (at density $=1.3$ ).

Secondly, the experiments considered 3Regular Graphs. In this case the randomly generated instances have an even number of nodes with constant density 1.5. We found success probability to worsen with the number of nodes achieving roughly the same value of 0.07 for nodes $n=20$ (at density $=1.5$ ).

In both cases we find the considered instances appear on the edge of a fall-off region in our analysis of the density dependent performance landscape. It is for instances beyond these densities (that is, beyond critical approximation density fall off) that we observe limited performance for depth three QAOA ansatz.

Finally, the mean field Sherrington-Kirkpatrick (SK) model is considered where the generated 
graph instances are fully connected and have the maximum possible density $((n-1) / 2)$ for $n$ nodes ranging from 10 to 20 .

Unlike the previous two cases, the SK instances scale up density linearly with $n$. Furthermore, for any fixed $n$, such graph instances have the highest density and therefore QAOA should indicate poor performance. This agrees exactly with our emulation. For each $n$, we observe the success probability for the SK model to be the lowest in the considered family of graphs (see the bottom pane on Fig. 1).

\section{Methods}

\subsection{QAOA}

Given an objective function which encodes the solution to an optimization problem in the ground state of a Hamiltonian, $\mathcal{V}$, the usual QAOA procedure is as follows,

1. Generate ansatz states, $|\psi(\boldsymbol{\gamma}, \boldsymbol{\beta})\rangle$ on a quantum computer, where $\gamma=\left(\gamma_{1}, \gamma_{2}, \cdots, \gamma_{p}\right)$ and $\boldsymbol{\beta}=\left(\beta_{1}, \beta_{2}, \cdots, \beta_{p}\right)$ are tunable real parameters over some fixed range. The state is prepared by applying a sequence of $2 p$ parameter unitary gates acting on the reference state $|+\rangle^{\otimes n}$ which is the symmetric superposition of all $2^{n}$ computational basis states as follows,

$$
|\psi(\boldsymbol{\gamma}, \boldsymbol{\beta})\rangle=\prod_{k=1}^{p} \mathcal{U}\left(\gamma_{k}, \beta_{k}\right)|+\rangle^{\otimes n},
$$

where

$$
\mathcal{U}\left(\gamma_{k}, \beta_{k}\right)=\exp \left(-i \beta_{k} \mathcal{H}_{x}\right) \cdot \exp \left(-i \gamma_{k} \mathcal{V}\right)
$$

The Hamiltonian $\mathcal{H}_{x}=\sum_{i} \sigma_{x}^{(i)}$ is called the driver Hamiltonian, and $\mathcal{V}$ is the problem Hamiltonian here ground states correspond to the bit strings that minimizes the objective function of interest.

2. Measurement of the state in Eq. (1) is done to compute the expected value, $\langle\psi(\boldsymbol{\gamma}, \boldsymbol{\beta})|\mathcal{V}| \psi(\boldsymbol{\gamma}, \boldsymbol{\beta})\rangle$. This is an approximation which can be calculated efficiently on a classical computer.

3. Steps 1 and 2 are repeated and a classical optimization algorithms is used to assign a set of optimum parameters, $\gamma^{*}$ and $\boldsymbol{\beta}^{*}$ that minimize $\langle\psi(\boldsymbol{\gamma}, \boldsymbol{\beta})|\mathcal{V}| \psi(\boldsymbol{\gamma}, \boldsymbol{\beta})\rangle$. $\left\langle\psi\left(\boldsymbol{\gamma}^{*}, \boldsymbol{\beta}^{*}\right)|\mathcal{V}| \psi\left(\boldsymbol{\gamma}^{*}, \boldsymbol{\beta}^{*}\right)\right\rangle$ then represents an approximate solution to the optimization problem.

\subsection{Problem description}

In this paper we study minimization problem on graphs. The objective function of interest can be defined as follows:

Given an undirected weighted graph $G=$ $(V, E)$, with $|V|=n$ nodes and $|E|=m$ edges. $E$ denotes the set of edges in $G$ as $E=\{(i, j)\}$, where $i \neq j$ and $i, j \in V$. In general, the edge weights are defined as a map $w: E \rightarrow \mathbb{R}$. Yet in our case and without loss of generality, we restrict to sampling $w_{i j}$ s from a uniform distribution over $\{-1,+1\}$.

Minimization problems on $G$ are defined thorough a cost-function $\mathcal{C}(\boldsymbol{z})$ with a corresponding embedding as quantum Hamiltonian operator. Here we employ the following as our objective function,

$$
\mathcal{C}=\sum_{(i, j) \in E} w_{i j} Z_{i} Z_{j}
$$

where $\boldsymbol{z}$ denotes the bitstring or bit assignment to the nodes in $G$ and $Z_{i}$ corresponds to the Pauli $Z$ operator action on the $i^{\text {th }}$ qubit. In general, with appropriate cost-function construction and Ising spin encoding, minimization on graphs can represent solving a family of NP-hard optimization problems such as MAX-CUT, Vertex cover and Maximum Independent Set to name a few.

\subsection{Performance metrics}

QAOA performance is analysed under the following two performance metrics,

1. the error in best possible approximation,

2. the success probability or the ground state overlap.

Let $|\psi\rangle$, be the $p$-depth QAOA ansatz states generated by Eq. (1) and let $\mathcal{A}$ be a subset that is not nessisarily equal to $\mathbb{C}_{2}^{\otimes n}$. Then we define the error in best possible approximation as

$$
f=\min _{|\psi\rangle \in \mathcal{R} \subset \mathbb{C}_{2}^{\otimes n}}\langle\psi|\mathcal{C}| \psi\rangle-\min _{|\phi\rangle \in \mathbb{C}_{2}^{\otimes n}}\langle\phi|\mathcal{C}| \phi\rangle .
$$

This quantity characterises the limiting performance of QAOA. The first term on the right indicates minimization over the set of reachable states 
generated by a fixed depth QAOA ansatz whereas the second term represents the exact optimum value of $\mathcal{C}$ computed by minimization over the entire Hilbert space.

To calculate the success probability or the ground state overlap, let $\left\{\left|\mathrm{gs}_{i}\right\rangle\right\}$ be the set of $d$ degenerate ground states of $\mathcal{C}$ then the overlap is given by,

$$
\eta=\sum_{i=1}^{d}\left|\left\langle\psi_{p}(\boldsymbol{\gamma}, \boldsymbol{\beta}) \mid \mathrm{gs}_{i}\right\rangle\right|^{2}
$$

A well known performance metric is the approximation ratio defined as,

$$
r=\frac{\langle\mathcal{C}\rangle}{\mathcal{C}_{\min }}
$$

It is under this performance metric that Google's data are represented [1]. Although this metric is bounded between $[0,1]$, and it can directly be used to contrast performance with other polynomial-time approximation algorithms, we observe the effect of density dependence to be visually suppressed. This is due to the logistic behaviour of the error in best possible approximation, $\langle\mathcal{C}\rangle-\mathcal{C}_{\text {min }}$. Since both $\langle\mathcal{C}\rangle$ and $\mathcal{C}_{\text {min }}$ grow with density for any given $n$ and fixed depth QAOA ansatz, the ratio visually conceals density dependence. This is further illustrated when we consider success probability as our performance indicator. Under this choice we clearly observe the limiting performance of QAOA.

\subsection{Graph Instances}

Here we present the construction of random problem instances used in our work.

Fixed $n$ case. We first show the approximation inefficiency or the feature of density dependent performance under the two metrics by studying QAOA on the uniform random graph model, $G_{n, m}$ for fixed $n$. The density in the case of such graph problems can be defined as the ratio of the number of edges to the number of nodes, $m / n$.

Random instances are generated by initializing an empty graph on the vertex set of size $|V|=n$. Then, $m$ edges are constructed in such a way that all possible $\left(\begin{array}{c}n \\ 2 \\ m\end{array}\right)$ choices are equally likely with random weights drawn from $\{-1,+1\}$.

3-regular graphs. A 3-regular graph, also known as a cubic graph, is when each node in the graph has degree equal to 3 . The density of generated instance in this case is constant $($ density $=1.5)$ as the number of edges required for constructing a 3-regular graph is given by $3 \times \frac{n}{2}$, for even $n$.

2-D grid graphs. These graphs are interesting mainly due to the inherent hardware connectivity of Google's Sycamore. Such graphs are generated by constructing an $N \times N$ square grid and randomly deleting nodes on the boundaries to construct random grid instance with $n \leq N^{2}$ nodes. Unlike the case of regular graphs, 2-D grid graphs do not share a constant density across the generated instance. The maximum number of edges in a square/partially square grid is given by $\lfloor 2 n-2 \sqrt{n}\rfloor$. Therefore, at most density of such instances is bounded above by 2.0 .

\subsection{Numerical Methods}

The large calculations were performed on Skotlech's Zhores computer [28]. Our simulation of QAOA is based on recovering the exact state vector in a noiseless setting. Therefore in calculation of success probability infinite sampling is assumed. Standard L-BFGS-B optimizer from SciPy with 25 random seed runs are used to find good optimal parameters to minimize the objective function. The procedure is then repeated over 100 randomly generated graph instances at each respective density to evaluate average performance and the corresponding standard deviation (represented in the plots as error-bars). The code is written in Python with Intel optimised libraries and is available on reasonable request.

\section{Conclusions}

To assess QAOA performance, Google applies approximation ratio - a standard technique in the classical theory of graph optimization (see e.g. [1]). Under this setting, an almost constant performance across the three graph problem families is visually observed - reproduced in Fig. 1 top pane.

It is only under other indicators such as (i) best approximation error and (ii) success probability - see Methods - that the density dependence in approximation inefficiency is visually revealed (a.k.a. reachability deficits). Although the effect can be inferred from Google's approximation ratio, the effect of reachability deficits are 
highly suppressed visually.

We finally assert that future experimental and numerical studies related to QAOA should report and contrast algorithmic performance with instance densities.

\section{Acknowledgments}

The authors acknowledge support from the project, Leading Research Center on Quantum Computing (Agreement No. 014/20). The authors acknowledge the use of Skoltech's Zhores supercomputer for obtaining the numerical results presented in this paper.

Competing interests. The authors declare no competing interests.

Author contributions. All authors conceived and developed the theory and design of this study and verified the methods. JB proposed the study and supervised its execution. VA, HP and IZ developed and deployed the parallel code to collect numerical data. All authors contributed to interpret the results and wrote the manuscript.

Data and code availability. The data that supports the findings of this study are available within the article. The code for generating the data is available on GitHub at https://github.com/vishwanathanakshay/QAOA.

\section{References}

[1] Matthew P Harrigan, Kevin J Sung, Matthew Neeley, Kevin J Satzinger, Frank Arute, Kunal Arya, Juan Atalaya, Joseph C Bardin, Rami Barends, Sergio Boixo, et al. Quantum approximate optimization of nonplanar graph problems on a planar superconducting processor. Nature Physics, 17(3): 332-336, 2021. DOI: 10.1038/s41567-02001105-y.

[2] Frank Arute, Kunal Arya, Ryan Babbush, Dave Bacon, Joseph C Bardin, Rami Barends, Rupak Biswas, Sergio Boixo, Fernando GSL Brandao, David A Buell, et al. Quantum supremacy using a programmable superconducting processor. Nature, 574 (7779):505-510, 2019. DOI: 10.1038/s41586019-1666-5.

[3] Alán Aspuru-Guzik, Anthony D Dutoi, Peter J Love, and Martin Head-Gordon. Simu- lated quantum computation of molecular energies. Science, 309(5741):1704-1707, 2005. DOI: $10.1126 /$ science.1113479.

[4] Peter JJ O'Malley, Ryan Babbush, Ian D Kivlichan, Jonathan Romero, Jarrod R McClean, Rami Barends, Julian Kelly, Pedram Roushan, Andrew Tranter, Nan Ding, et al. Scalable quantum simulation of molecular energies. Physical Review X, 6(3):031007, 2016. DOI: 10.1103/PhysRevX.6.031007.

[5] Jacob Biamonte, Peter Wittek, Nicola Pancotti, Patrick Rebentrost, Nathan Wiebe, and Seth Lloyd. Quantum machine learning. Nature, 549(7671):195-202, 2017. DOI: 10.1038 /nature23474.

[6] Rongxin Xia and Sabre Kais. Quantum machine learning for electronic structure calculations. Nature communications, 9(1):1-6, 2018. DOI: $10.1038 / \mathrm{s} 41467-018-06598-z$.

[7] Seth Lloyd. Universal quantum simulators. Science, pages 1073-1078, 1996. URL https://www.jstor.org/stable/ 2899535.

[8] Lucas Lamata, Antonio Mezzacapo, Jorge Casanova, and Enrique Solano. Efficient quantum simulation of fermionic and bosonic models in trapped ions. EPJ Quantum Technology, 1(1):9, 2014. DOI: 10.1140/epjqt9.

[9] Tadashi Kadowaki and Hidetoshi Nishimori. Quantum annealing in the transverse ising model. Physical Review E, 58(5):5355, 1998. DOI: $10.1103 /$ PhysRevE.58.5355.

[10] Guido Pagano, Aniruddha Bapat, Patrick Becker, Katherine S Collins, Arinjoy De, Paul W Hess, Harvey B Kaplan, Antonis Kyprianidis, Wen Lin Tan, Christopher Baldwin, et al. Quantum approximate optimization of the long-range ising model with a trapped-ion quantum simulator. Proceedings of the National Academy of Sciences, 117(41):25396-25401, 2020. DOI: 10.1073 /pnas.2006373117.

[11] Edward Farhi, Jeffrey Goldstone, and Sam Gutmann. A quantum approximate optimization algorithm. arXiv preprint arXiv:1411.4028, 2014. URL https:// arxiv.org/abs/1411.4028.

[12] Jarrod R McClean, Jonathan Romero, Ryan Babbush, and Alán Aspuru-Guzik. The theory of variational hybrid quantum-classical algorithms. New Journal of Physics, 18 
(2):023023, 2016. DOI: $10.1088 / 1367-$ 2630/18/2/023023.

[13] Jacob Biamonte. Universal variational quantum computation. Phys. Rev. A, 103: L030401, Mar 2021. DOI: 10.1103/PhysRevA.103.L030401.

[14] Seth Lloyd. Quantum approximate optimization is computationally universal. arXiv preprint arXiv:1812.11075, 2018. URL https://arxiv.org/abs/1812.11075.

[15] Mauro ES Morales, Jacob D Biamonte, and Zoltán Zimborás. On the universality of the quantum approximate optimization algorithm. Quantum Information Processing, 19(9):1-26, 2020. DOI: 10.1007/s11128-02002748-9.

[16] Cedric Yen-Yu Lin and Yechao Zhu. Performance of QAOA on Typical Instances of Constraint Satisfaction Problems with Bounded Degree. arXiv e-prints, art. arXiv:1601.01744, January 2016. URL https://arxiv.org/abs/1601.01744.

[17] Gavin E Crooks. Performance of the quantum approximate optimization algorithm on the maximum cut problem. arXiv preprint arXiv:1811.08419, 2018. URL https:// arxiv.org/abs/1811.08419.

[18] Samuel Marsh and JB Wang. A quantum walk-assisted approximate algorithm for bounded np optimisation problems. Quantum Information Processing, 18(3):61, 2019. DOI: $10.1007 / \mathrm{s} 11128-019-2171-3$.

[19] Zhihui Wang, Stuart Hadfield, Zhang Jiang, and Eleanor G Rieffel. Quantum approximate optimization algorithm for maxcut: A fermionic view. Physical Review A, 97(2):022304, 2018. DOI: 10.1103/PhysRevA.97.022304.

[20] Zhang Jiang, Eleanor G Rieffel, and Zhihui Wang. Near-optimal quantum circuit for grover's unstructured search using a transverse field. Physical Review A, 95(6):062317, 2017. DOI: 10.1103/PhysRevA.95.062317.

[21] Mauro ES Morales, Timur Tlyachev, and Jacob Biamonte. Variational learning of grover's quantum search algorithm. Physical Review A, 98(6):062333, 2018. DOI: 10.1103/PhysRevA.98.062333.

[22] V. Akshay, H. Philathong, M. E. S. Morales, and J. D. Biamonte. Reachability deficits in quantum approximate optimization. Phys.
Rev. Lett., 124:090504, Mar 2020. DOI: 10.1103/PhysRevLett.124.090504.

[23] JS Otterbach, $\mathrm{R}$ Manenti, $\mathrm{N}$ Alidoust, A Bestwick, M Block, B Bloom, S Caldwell, N Didier, E Schuyler Fried, S Hong, et al. Unsupervised machine learning on a hybrid quantum computer. arXiv preprint arXiv:1712.05771, 2017. URL https:// arxiv.org/abs/1712.05771.

[24] Madita Willsch, Dennis Willsch, Fengping Jin, Hans De Raedt, and Kristel Michielsen. Benchmarking the quantum approximate optimization algorithm. Quantum Information Processing, 19:197, 2020. DOI: 10.1007/s11128-020-02692-8.

[25] Deanna M Abrams, Nicolas Didier, Blake R Johnson, Marcus P da Silva, and Colm A Ryan. Implementation of xy entangling gates with a single calibrated pulse. $\mathrm{Na}$ ture Electronics, 3(12):744-750, 2020. DOI: 10.1038/s41928-020-00498-1.

[26] Andreas Bengtsson, Pontus Vikstål, Christopher Warren, Marika Svensson, Xiu Gu, Anton Frisk Kockum, Philip Krantz, Christian Križan, Daryoush Shiri, Ida-Maria Svensson, et al. Improved success probability with greater circuit depth for the quantum approximate optimization algorithm. Physical Review Applied, 14(3):034010, 2020. DOI: 10.1103/PhysRevApplied.14.034010.

[27] Xiaogang Qiang, Xiaoqi Zhou, Jianwei Wang, Callum M Wilkes, Thomas Loke, Sean O'Gara, Laurent Kling, Graham D Marshall, Raffaele Santagati, Timothy C Ralph, et al. Large-scale silicon quantum photonics implementing arbitrary two-qubit processing. Nature photonics, 12(9):534-539, 2018. DOI: $10.1038 / \mathrm{s} 41566-018-0236-y$.

[28] Igor Zacharov, Rinat Arslanov, Maksim Gunin, Daniil Stefonishin, Andrey Bykov, Sergey Pavlov, Oleg Panarin, Anton Maliutin, Sergey Rykovanov, and Maxim Fedorov. "zhores" - petaflops supercomputer for data-driven modeling, machine learning and artificial intelligence installed in skolkovo institute of science and technology. Open Engineering, 9(1):512-520, October 2019. DOI: 10.1515/eng-2019-0059. 

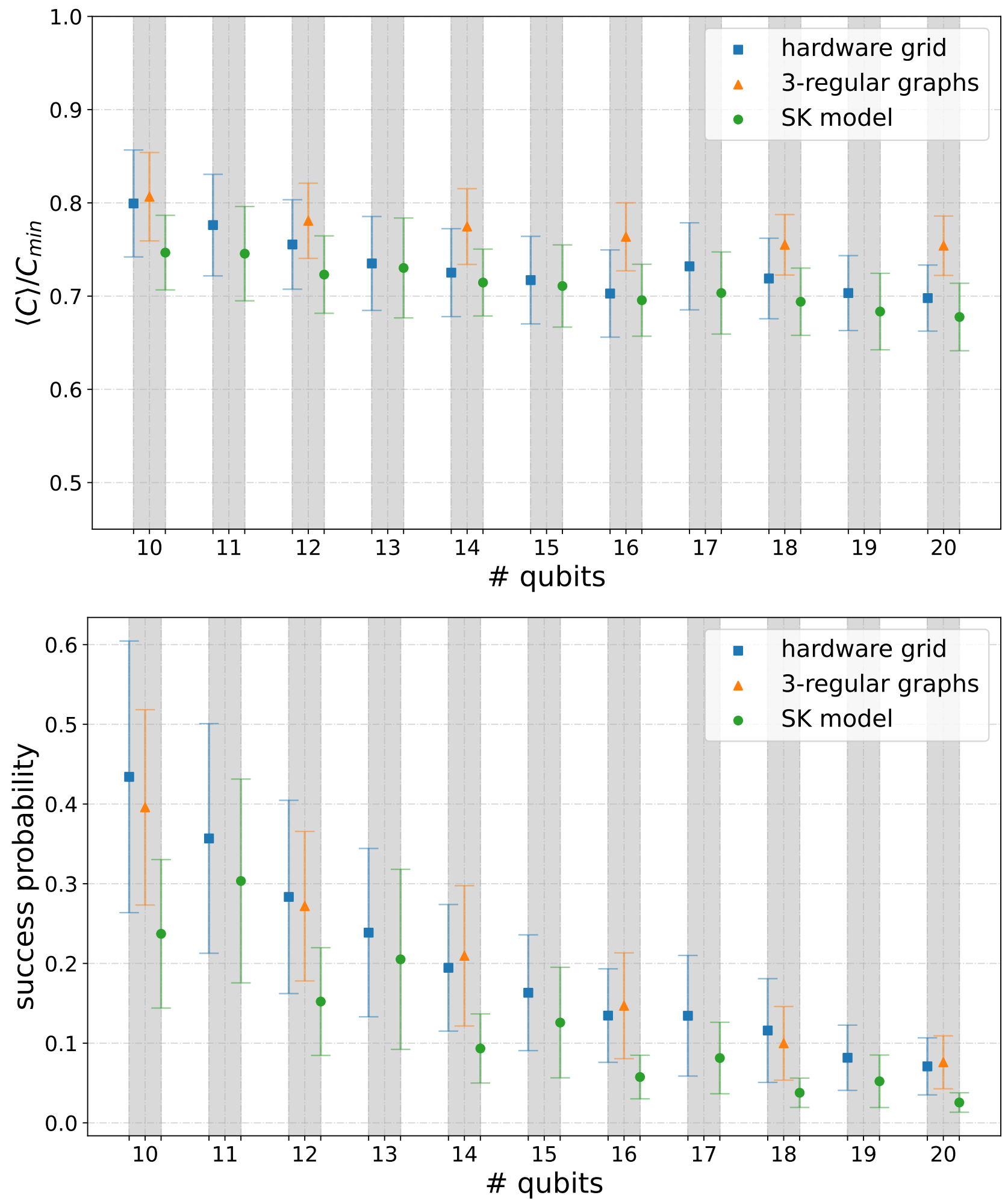

Figure 1: Reproducing Google's ideal noiseless data for the three families of graph, (i) hardware grid graphs (blue), (ii) 3-regular graphs (orange), and (iii) SK model or complete graphs (green). Each data point represents the average performance of depth $p=3$ QAOA over statistics of 100 randomly generated instances. We observe that the effect of density dependence in QAOA performance is clearly observed when considering the success probability as the metric (bottom). On other hand, it remains visually suppressed using Google's approximation ratio (top). 

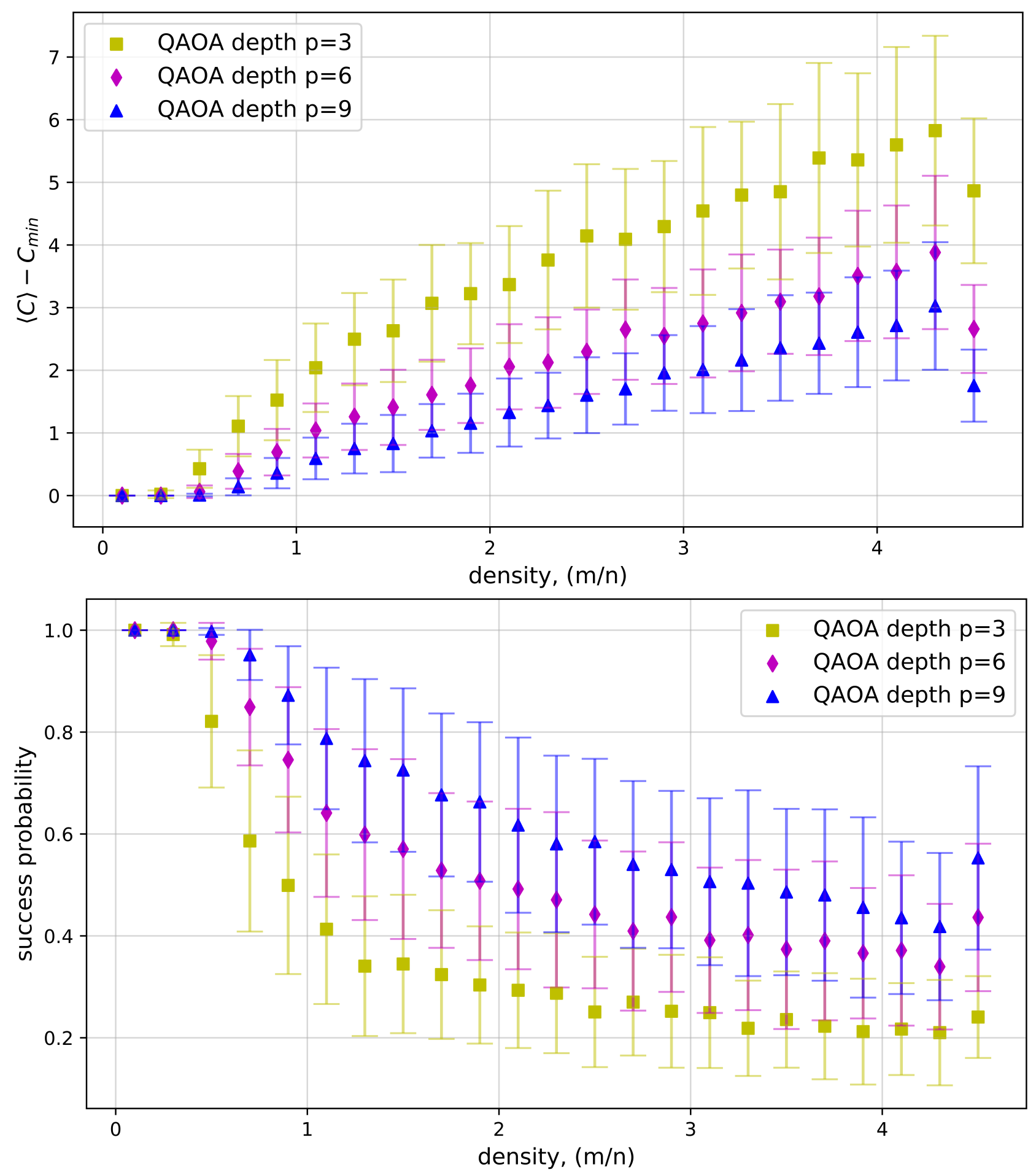

Figure 2: QAOA performance metric as a function of density for depth $p=3,6,9$; (top) error in best possible approximation, and (bottom) success probability or ground state overlap. Each data point represents the average performance over statistics of 100 uniform random graph instances, $G_{n, m}$ with nodes $n=10$. Here we see the strong correlation between approximation inefficiency and density in both performance metrics. 

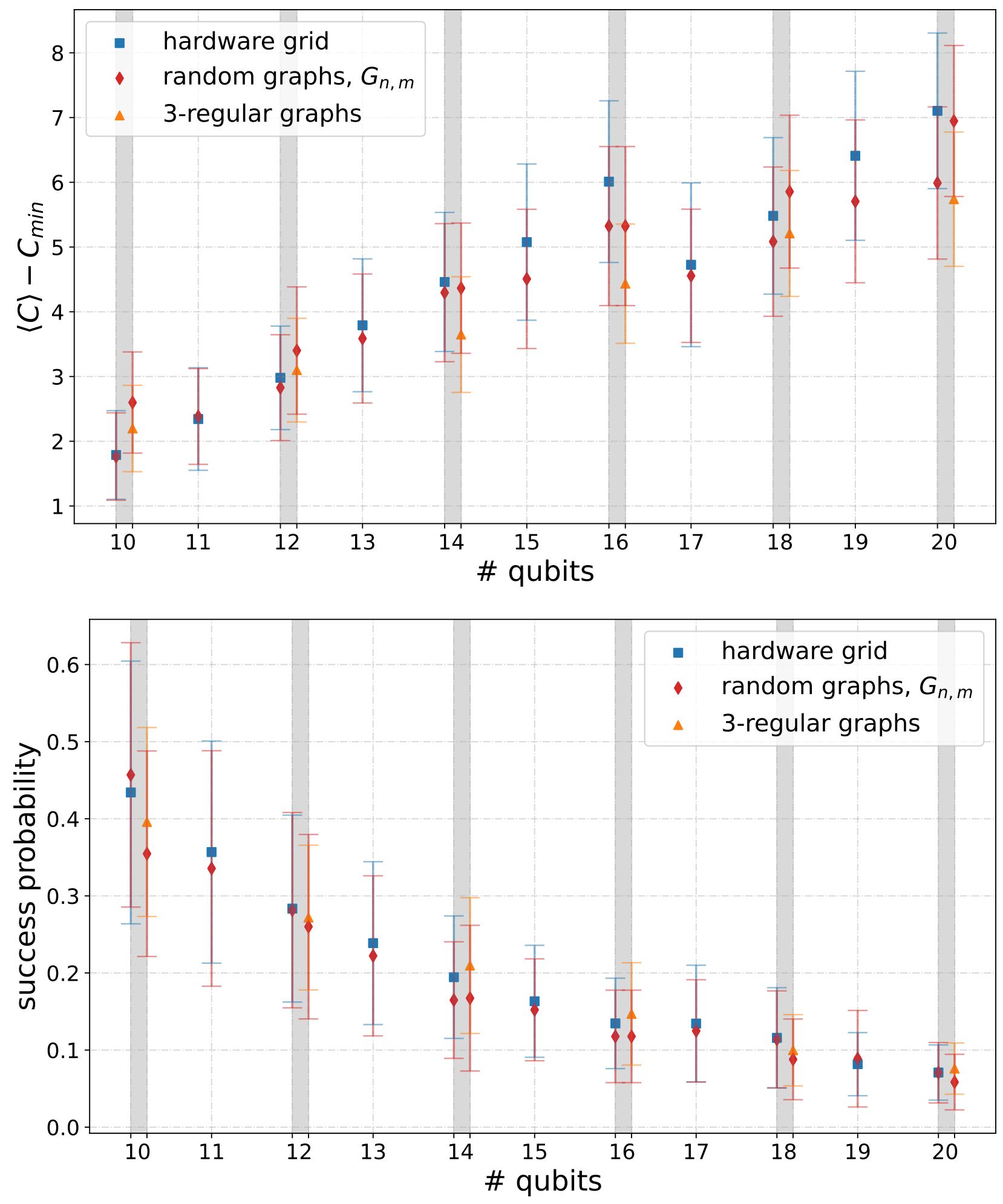

Figure 3: Comparing (top) error in best possible approximation and (bottom) success probability of the three graph families. For even numbers of qubits, the left vertical pair represents the grid to random graph comparison, and the right vertical pair represents the 3-regular to random graph comparison. For odd numbers of qubits, the grid to random graph comparison is shown (since 3-regular graphs can be generated only with even number of nodes). Each data point represents the average performance of depth $p=3$ QAOA over statistics of 100 randomly generated instances. By comparison, we do not observe any topology related bias in QAOA performance that can be of statistical relevance. 

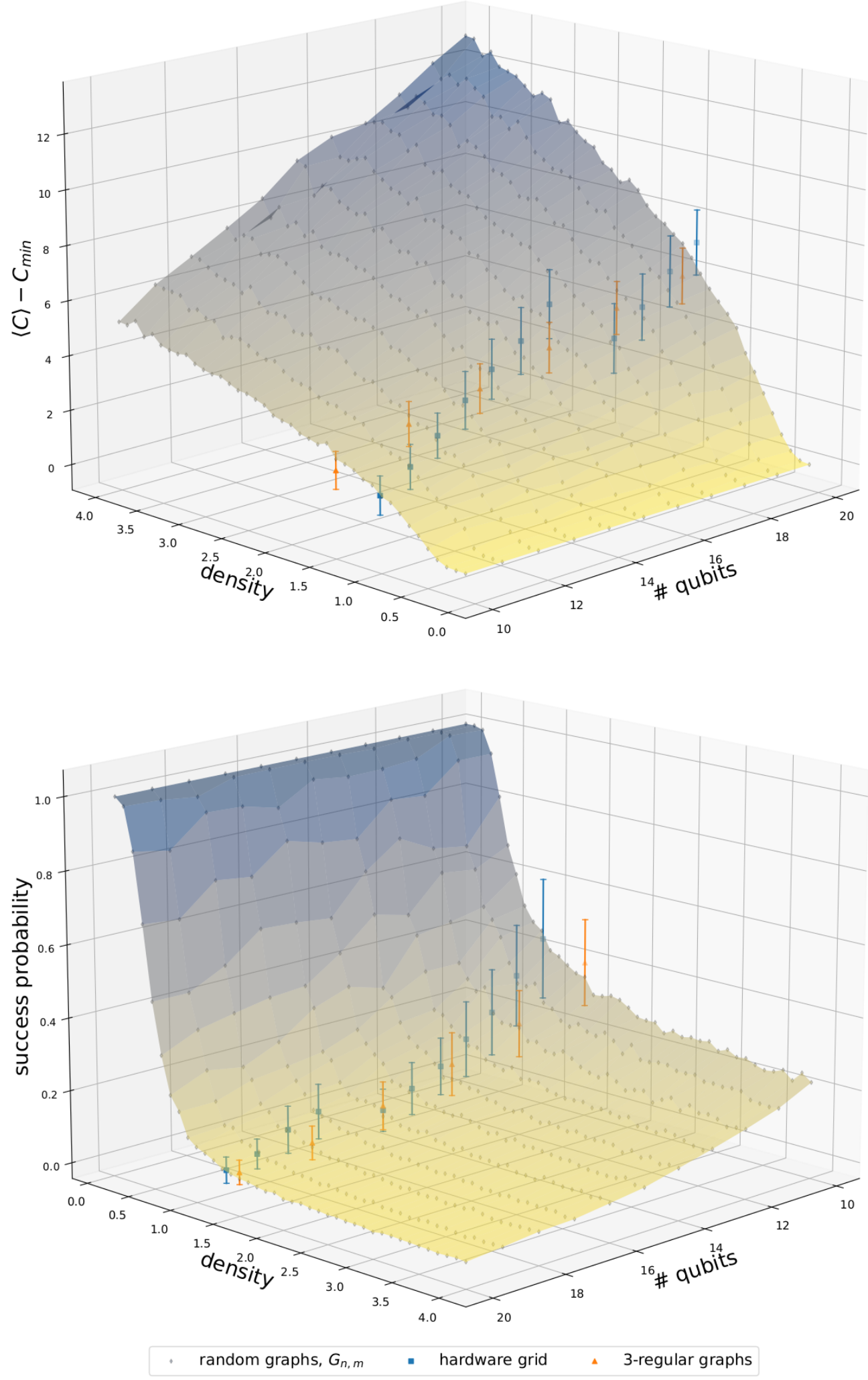

Figure 4: 3D landscape of depth-3 QAOA performances; (top) error in best possible approximation and (bottom) success probability. Google data points are marked on the landscape according to the mean density and performance of the considered instances. We observe a sharp fall-off behaviour in QAOA performance which appears to saturate logistically beyond intermediate density. We find that Google data points lie between densities 1 and 1.5, where a low depth QAOA ansatz can approximate a solution(s) efficiently. 

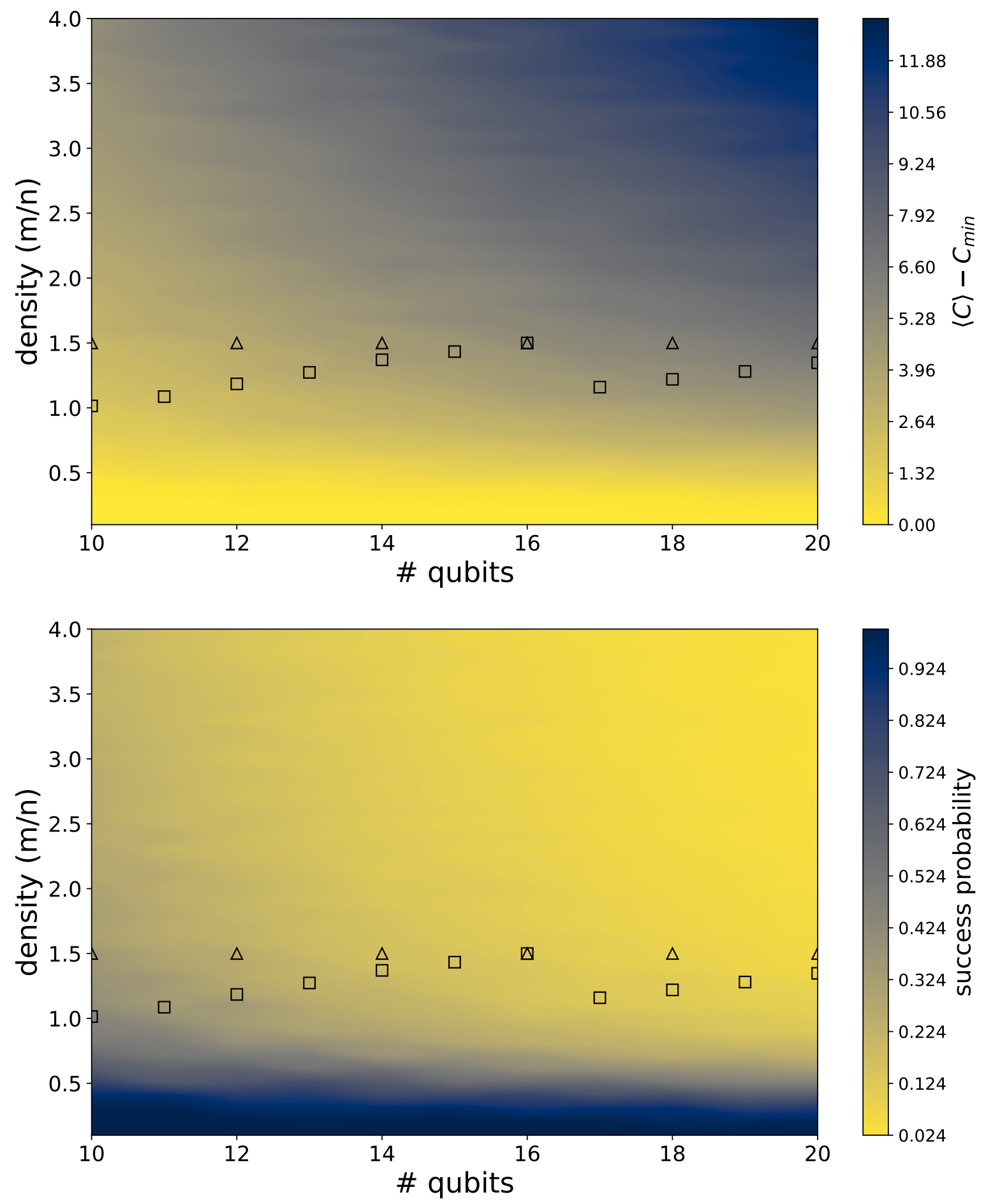

Figure 5: 2D projection of depth-3 QAOA performance; (top) error in best possible approximation and (bottom) success probability. Google data points are marked on the landscape according to the mean density of the considered instances. Squares represent grid instances and Triangles represent 3 regular graphs. Google data points lie between density 1 and 1.5, where a low depth QAOA ansatz can approximate a solution(s) efficiently. 